\title{
CDMA-Based MAC Protocol for Wireless Ad Hoc Networks *
}

\author{
Alaa Muqattash and Marwan Krunz \\ Department of Electrical and Computer Engineering \\ The Unniversity of Arizona \\ Tucson, Arizona 85719 \\ alaa,krunz@ece.arizona.edu
}

\begin{abstract}
We propose a CDMA-based power controlled medium access protocol for mobile ad hoc networks (MANETs). Unlike previously proposed protocols, ours accounts for the multiple access interference (MAI), thereby addressing the notorious near-far problem that undermines the throughput performance in MANETs. Channel-gain information obtained from overheard RTS and CTS packets over an outof-band control channel is used to dynamically bound the transmission power of mobile terminals in the vicinity of a receiver. By properly estimating the required transmission power for data packets, the proposed protocol allows for interference-limited simultaneous transmissions to take place in the neighborhood of a receiving terminal. Simulation results indicate that compared to the IEEE 802.11 approach, the proposed protocol achieves a significant increase in network throughput at no additional cost in energy consumption.
\end{abstract}

\section{Categories and Subject Descriptors}

C.2.1 [Network Architecture and Design]: Wireless Communication; C.2.2 [Network Protocols]

\section{General Terms}

Algorithm, Design, Performance

\section{Keywords}

Ad hoc networks, CDMA, multi-access interference, near-far problem, power control, code assignment.

\footnotetext{
*This work was supported in part by the National Science Foundation under grants ANI 9733143, CCR 9979310, and ANI 0095626, and by the Center for Low Power Electronics (CLPE) at the University of Arizona (Grant \# EEC9523338).
}

Permission to make digital or hard copies of all or part of this work for personal or classroom use is granted without fee provided that copies are not made or distributed for profit or commercial advantage and that copies bear this notice and the full citation on the first page. To copy otherwise, to republish, to post on servers or to redistribute to lists, requires prior specific permission and/or a fee.

MobiHoc'03, June 1-3, 2003, Annapolis, Maryland, USA.

Copyright 2003 ACM 1-58113-684-6/03/0006 ...\$5.00.

\section{INTRODUCTION}

\subsection{Motivation}

Mobile ad hoc networks (MANETs) have recently been the topic of extensive research. The interest in such networks stems from their ability to provide a temporary wireless networking capability in scenarios where fixed infrastructures are lacking and are expensive or infeasible to deploy (e.g., disaster relief efforts, battlefields, etc.). While wide deployment of MANETs is yet to come, many efforts are currently underway to standardize protocols for the operation and management of such networks [26, 2].

One of the fundamental challenges in MANETs research is how to increase the overall network throughput while maintaining low energy consumption for packet processing and communications. The low throughput is attributed to the harsh characteristics of the radio channel combined with the contention-based nature of medium access control (MAC) protocols commonly used in MANETs. The focus of this paper is on improving the network throughput of a MANET by means of a CDMA-based design of the MAC protocol. Compared to the DCF (Distributed Coordination Function) mode of the IEEE 802.11 standard [3], which is currently the de facto MAC protocol for MANETs, our MAC protocol is shown to achieve a significant increase in network throughput for the same or less energy consumption per delivered packet.

CDMA is based on spread spectrum (SS) techniques, in which each user occupies the entire available bandwidth. At the transmitter, a digital signal of bandwidth, say $B_{1}$ bits/sec, is spread using (i.e., multiplied by) a pseudo-random noise $(\mathrm{PN})$ code of bandwidth, say $B_{2} \mathrm{bits} / \mathrm{sec}\left(B_{2} / B_{1} \gg 1\right.$ is called the processing gain). The $\mathrm{PN}$ code is a binary sequence that statistically satisfies the requirement of a random sequence, but that can be exactly reproduced at the intended receiver through precise mathematical rules. Using a locally generated $\mathrm{PN}$ code, the receiver de-spreads the received signal, recovering from it the original information. The enhancement in performance obtained from spreading the signal makes it possible for several, independently coded signals to occupy the same channel bandwidth, provided that each signal has a distinct PN code. This type of communication in which each transmitter-receiver pair has a distinct $\mathrm{PN}$ code for transmitting over a common channel is called CDMA [24].

Due to its superior characteristics, CDMA has been the 
access technology of choice in cellular systems, including the recently adopted $3 \mathrm{G}$ systems [22]. In such systems, CDMA has been shown to provide up to six times the capacity of TDMA- or FDMA-based solutions [12]. This throughput gain comes along with other desirable features, including graceful signal degradation, multipath resistance, inherent frequency diversity, and interference rejection. It is, therefore, of no surprise that CDMA is being considered for ad hoc networks. Interestingly, the IEEE 802.11 standard uses SS techniques at the physical layer ${ }^{1}$, but only to mitigate the impact of the harsh wireless channel. More specifically, in the 802.11 protocol all transmitted signals are spread using a common PN code, precluding the possibility of multiple concurrent transmissions in the a vicinity of a receiver. This situation is exemplified in Figure 1, where the transmissions $A \rightarrow B$ and $C \rightarrow D$ cannot take place at the same time.

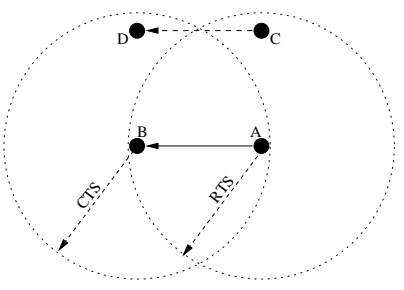

Figure 1: Example showing the low throughput of the 802.11 scheme (only one transmission can take place at a time).

\subsection{Code Assignment and Spreading Protocol Issues}

Enabling CDMA-based solutions for MANETs is fraught with challenges, which are essentially related to the absence of centralized control (i.e., a base station). First, a code assignment protocol is needed to assign distinct codes to different terminals. This problem is trivial in small networks, but becomes acute in large networks where the number of $\mathrm{PN}$ codes is smaller than the number of terminals ${ }^{2}$, necessitating spatial reuse of the PN codes. Several code assignment protocols have been previously proposed (e.g., [14, 4, 11]). In general, these protocols attempt to assign codes to nodes with the constraint that all neighbors of a node have different PN codes [14].

Besides the code assignment protocol, a spreading-code protocol is also needed to decide which codes to use for packet transmission and for monitoring the channel in anticipation of a packet reception [30]. Such a protocol can be receiverbased, transmitter-based, or a hybrid. In a receiver-based protocol, the transmitter uses the code of the intended receiver to spread the packet, while an idle terminal constantly monitors its own code. This approach simplifies the re-

\footnotetext{
${ }^{1}$ Both direct sequence spread spectrum (DSSS) and frequency hopping spread spectrum (FHSS) are included in the IEEE 802.11 specifications. However, since DSSS has more desirable properties than FHSS, it has been favored in recent wireless standards, including IS-95. Accordingly, our focus in this paper is on DSSS techniques.

${ }^{2}$ The number of codes is usually constrained by the available spectrum and the required information data rate [29].
}

ceiver's circuitry because the receiver does not have to monitor the whole code set. Unfortunately, primary collisions are still possible, even under a correct code assignment (a primary collision involves two or more transmissions that are spread using the same code). For example, consider two non-neighboring nodes $A$ and $C$ that have two different codes. These nodes may have a common neighbor, say $B$, with its own code. A primary collision may occur if nodes $A$ and $C$ simultaneously attempt to transmit to node $B$ using $B$ 's code $^{3}$. The only way to guarantee that primary collisions cannot happen is to use different codes for different, concurrently transmitted signals (not nodes). Another disadvantage of the receiver-based approach is that a broadcast requires the transmitter to unicast the message to each receiver. In a transmitter-based spreading protocol, a transmission code is assigned to each terminal, and receivers must be able to monitor the activity on the whole set of PN codes. The advantage of this approach is that primary collisions cannot happen. In addition, broadcast is inherently supported. However, the drawback is that the receiver circuitry is very complex and expensive. Various hybrids of the above two approaches are also possible. For example, the authors in [30] proposed two hybrid schemes: the commontransmitter-based protocol and the receiver-transmitter-based protocol. In the first protocol, the fields in the packet header that contain the source and destination addresses are spread using a common code, while the rest of the packet is spread using the transmitter's code. An idle terminal constantly monitors the common code. Upon recognizing its address in the destination field, the listening terminal switches to the code of the transmitting node to receive the rest of the packet. The receiver-transmitter-based works similarly, but with the common code replaced with the receiver's code.

\subsection{Goals and Paper Contributions}

Several CDMA-based MAC protocols for MANETs have been proposed in the literature (e.g., $[30,15,19,11,16])$. These protocols, in general, are based on random channel access, whereby a terminal with a packet to transmit can proceed immediately with its transmission (starting, possibly, with an RTS/CTS exchange), irrespective of the state of the channel. We refer to such schemes as random access CDMA (RA-CDMA). Under appropriate code assignment and spreading-code schemes, RA-CDMA protocols are guaranteed to be free of primary collisions. However, as explained in details in Section 2, the nonzero cross-correlations between different CDMA codes can induce multi-access interference (MAI), resulting in secondary collisions at a receiver (collisions between two or more transmissions that use different CDMA codes). In the literature, this problem is known as the near-far problem [23]. As shown in Section 2, the near-far problem can cause a significant reduction in network throughput, and hence cannot be overlooked when designing $C D M A$-based MAC protocols for MANETs. Accordingly, the main goal of this paper is to provide a CDMA-based MAC solution for MANETs that addresses the near-far problem. In our protocol, the transmission powers are dynamically adjusted such that the MAI at any receiver is not strong enough

\footnotetext{
${ }^{3}$ Note, however, that if the received power of one signal is much greater than the other, then capture is still possible and the stronger signal can still be received correctly.
} 
to cause a secondary collision. As indicated in our simulations, this results in a significant improvement in network throughput at no additional cost in energy consumption. In fact, the proposed protocol is shown to achieve some energy saving compared to the 802.11 scheme. To the best of our knowledge, this is the first attempt to address the near-far problem in the design of MAC protocols for MANETs.

The rest of the paper is organized as follows. In Section 2, we explain the near-far problem in detail and show its adverse effect on the throughput performance. Section 3 provides an overview of related CDMA-based protocols for MANETs. The proposed protocol is presented in Section 4, followed by simulation results and discussion in Section 5 . Finally, our main conclusions are drawn in Section 6.

\section{THE NEAR-FAR PROBLEM IN RA-CDMA}

\subsection{Imperfect Orthogonality of CDMA Codes}

The roots of the near-far problem lies in the fact that unlike FDMA and TDMA channels which can be completely orthogonal, CDMA codes suffer from nonzero cross-correlation between codes. When a CDMA receiver de-spreads a signal, it effectively computes the cross-correlation between the signal and a locally generated $\mathrm{PN}$ sequence. If this $\mathrm{PN}$ sequence is identical to the one used to spread the signal at the transmitter (i.e., the message is intended to this receiver), cross-correlation computations restore the original information data. Otherwise, such computations result in either a zero or a nonzero value, depending on whether the system is synchronous or asychnronous.

A system is called time-synchronous if all signals originate from the same transmitter, as in the case of the downlink of a cellular CDMA network ${ }^{4}$. In here, synchrony is manifested in two ways. First, different transmissions that are intended for different receivers will have a common time reference. Second, from the viewpoint of a given mobile terminal, all signals (intended or not) propagate through the same paths, and thus suffer the same time delays. In synchronous systems, it is possible to design completely orthogonal spreading codes. In fact, in the IS-95 standard for cellular CDMA networks [24], each user of the channel is assigned a Hadamard (or Walsh) code. These codes are orthogonal and are used to "channelize" the available bandwidth.

On the other hand, a system is called time-asynchronous if signals originate from multiple transmitters, as in the case of the uplink of cellular networks and also in MANETs. The reasons behind the naming are twofold. First, since signals originate from different transmitters, it is generally not feasible to have a common time reference for all the transmissions that arrive at a receiver. Second, these transmissions propagate through different paths; thus, they suffer different time delays [25]. In an asynchronous system, it is not possible to design spreading codes that are orthogonal for all time offsets [24]. In this case, the cross-correlation between codes cannot be neglected. In fact, codes that are orthogonal in synchronous systems (e.g., Hadamard codes) exhibit high cross-correlation when not perfectly synchronized. In-

\footnotetext{
${ }^{4}$ Mathematically, it is possible to have multiple transmitters and have a synchronous system. However, in practice, it is difficult to achieve perfect synchronization between those transmitters.
}

stead, PN codes that are designed specifically to have low cross-correlation are used.

While the code design problem is crucial in determining the system performance, of greater importance is the problem of nonzero cross-correlation of the PN codes [23]. Unintended transmissions add nonzero MAI during the despreading at a receiver. The near-far problem is a severe consequence of MAI, whereby a receiver who is trying to detect the signal of the $i$ th transmitter may be much closer in distance to, say, the $j$ th transmitter than the $i$ th transmitter. When all transmission powers are equal, the signal from the $j$ th transmitter will arrive at the receiver in question with a sufficiently larger power than that of the $i$ th transmitter, causing incorrect decoding of the $i$ th transmission (i.e., a secondary collision).

\subsection{Impact of the MAI Problem}

We now elaborate on the performance implications of the MAI problem. Consider the reception of a packet at terminal $i$. Let $P_{0}^{(i)}$ be the average received power of the desired signal at the $i$ th terminal. Suppose that there are $K$ interfering transmissions with received powers $P_{j}, j=1, \ldots, K$. The quality of the intended reception is adequately measured by the effective bit energy-to-noise spectral density ratio at the detector, denoted by $\mu^{(i)}$. For an asynchronous directsequence BPSK system, $\mu^{(i)}$ is given by $[31,27]^{5}$ :

$$
\mu^{(i)} \triangleq \frac{E_{b}}{N_{0 \mathrm{eff}}}=\left(\frac{2 \sum_{j=1}^{K} P_{j}}{3 W P_{0}^{(i)}}+\frac{1}{\mu_{0}}\right)^{-1}
$$

where $W$ is the processing gain and $\mu_{0}$ is the $E_{b} / N_{0 \text { eff }}$ ratio at the detector in the absence of interference. As the interfering power increases, $\mu^{(i)}$ decreases, and the bit error probability increases. As an example, consider a CDMA system that uses BPSK modulation and a convolutional code with rate $1 / 2$, constraint length 7 , and soft decision Viterbi decoding. Let $W=100$. To achieve a bit error probability of $10^{-6}$, the required $E_{b} / N_{0 \text { eff }}$ is $5.0 \mathrm{~dB}$ [24]. Ignoring the thermal noise and using (1), the total interference power must satisfy:

$$
\frac{\sum_{j=1}^{K} P_{j}}{P_{0}^{(i)}} \leq 47.43
$$

Transmitters are, in general, situated at different distances from the receiver. Suppose that the transmission powers are fixed and equal. Consider the case of one interferer $(K=1)$ at distance $d_{1}$ from the receiver. Let $d_{0}$ be the distance between the receiver and the intended transmitter. Using the two-ray propagation model for terrestrial communications (power loss $\sim 1 / d^{4}$ ), it is easy to show that to satisfy the required bit error rate, we must have $d_{1} \geq 0.38 d_{0}$. So if there is only one interferer that is at distance less than $0.38 d_{0}$ from the receiver, reliable communication will not be possible (i.e., a secondary collision will occur).

\footnotetext{
${ }^{5}$ Assuming truly random sequences of a rectangular chip pulses and using a Gaussian approximation with constant but unequal powers.
} 
The above example shows that the near-far problem can severely affect packet reception, and consequently, network throughput. A good measure of network throughput is given by the expected forward progress (EFP) per transmission, defined as the product of the local throughput of a terminal and the distance between the transmitter and the receiver [31]. The EFP was derived in [31] for multihop RA-CDMA networks, assuming a slotted system and Poisson distributed terminals in the $2 \mathrm{D}$ space. Let $p$ be the probability that a terminal is transmitting a packet in a given time slot (i.e., the per-node load) and let $L$ be the number of nodes that are within a circle centered at the transmitter and of radius that equals the transmitter-receiver separation distance. A scaled version of the EFP is plotted in Figure 2 as a function of $p$ for various values of $L$. The figure shows that the EFP initially increases with $p$ up to some point, say $p^{*}$, beyond which the EFP starts to decrease rapidly with $p$. This says that the channel becomes unstable when the load exceeds $p^{*}$, which is caused by the increase in the number of transmitted packets beyond the multiple access capability of the system. Our goal is to design a CDMA-based MAC protocol that prevents this rapid degradation in network throughput.

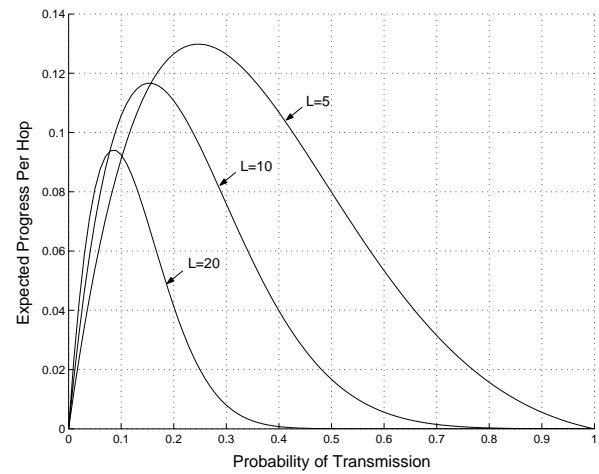

Figure 2: Throughput performance versus load in RA-CDMA networks.

\section{RELATED WORK}

In [30] the addresses part of the packet are spread using the common code, while the rest of the packet is spread using the transmitter-based approach. A receiver notes the address of the source terminal and uses this address to switch to the corresponding code. In [15] the authors proposed the coded tone sense protocol, in which $K$ busy tones are associated with $K$ spreading codes. During packet reception on a certain code, the receiving station broadcasts the corresponding busy tone. In [11] all terminals send the RTS-CTS packets on a common code, while the data packet are sent using a transmitter- or a receiver-based approach. Somewhat similar approaches were proposed in [16] and [34]. In all the above protocols, the authors assume perfect orthogonality between spreading codes, i.e., they ignore the near-far problem.

A reservation-based scheme was proposed in [33], whereby small control packets are used to request slot assignments for data packets. The authors investigated the use of FHSS to avoid MAI. Their approach, however, cannot be used for
DSSS, which is the method of choice in recent wireless standards (e.g. IS-95).

In [6] and [10] the authors proposed distributed channel assignment algorithms for SS multihop networks. Those protocols, however, do not allow for any MAI, and hence cannot support concurrent transmissions of signals with different codes. Clustering as proposed in [18] is another interesting approach for power control in CDMA networks. It simplifies the forwarding function for most terminals, but at the expense of reducing network utilization (since all communications have to go through the cluster heads). This can also lead to the creation of bottlenecks.

In [28] the authors proposed the use of a multiuser detection circuit at the receiver to mitigate the near-far problem in MANETs. The proposed scheme also requires the use of GPS receivers to provide accurate position and timing information. Such a scheme relies heavily on physical layer techniques to mitigate MAI, and makes no effort to account for MAI at the MAC layer. Moreover, although it is feasible to deploy multiuser GPS receivers at the base station, presently it is impractical (and expensive) to implement such receivers within the mobile terminal. Recently, an interesting approach for joint scheduling and power control in ad hoc networks was proposed [9]. This approach, however, requires a central controller for executing the scheduling algorithm, i.e., it is not a truely distributed solution. Furthermore, it assumes the existence of a separate feedback channel that enables receivers to send their SNR measurements to their respective transmitters in a contention free manner.

In [5] and [8] the authors analyzed RA-CDMA protocols for MANETs in the presence of MAI. They assumed that transmissions of all neighbors produce the same noise effect, and therefore, the SNR threshold can be converted into a threshold on the number of transmissions $(n)$ in the receiver's neighborhood. A packet is correctly received when that number is less than the predetermined threshold $n$. Hence, the protocol was called CDMA/ $n$. Although such an approximation may not be accurate in topologies where nodes are not equally spaced, it shows that MAI can significantly degrade network performance.

\section{THE PROPOSED PROTOCOL}

\subsection{Protocol Intuition and Design Goals}

Before presenting the operational details of the protocol, it is constructive to first discuss how the near-far problem is being addressed in cellular networks and why the same solution cannot be extended to MANETs. In the uplink of a cellular CDMA system, the near-far problem is combated through a combination of open- and closed-loop power control, which ensures that each mobile terminal generates the same signal power at the base station. The base station monitors the received signal power from each terminal and instructs faraway terminals to increase their signal powers and closeby terminals to decrease theirs. Unfortunately, the same solution cannot be used in MANETs. To see why, consider the situation in Figure 3. Let $d_{i j}$ denote the distance between nodes $i$ and $j$. Suppose that $A$ wants to communicate with $B$ using a given code and $C$ wants to communicate with $D$ using a different code. Suppsoe that $d_{A B} \approx d_{C D}$, $d_{C B} \ll d_{A B}$, and $d_{A D} \ll d_{C D}$. Then, the MAI caused by $C$ 
makes it impossible for $B$ to receive $A$ 's transmission. Similarily, the MAI caused by $A$ makes it impossible for $D$ to receive $C$ 's transmission. It is important to note that the two transmissions cannot take place simultaneously, irrespective of what transmission powers are selected (e.g., if $A$ increases its power to combat the MAI at $B$, then this increased power will destroy the reception at $D$ ).

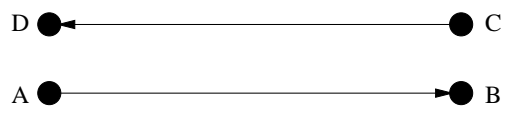

Figure 3: Example that demonstrates that power control alone is not enough to combat the near-far problem in MANETs.

The above example reveals two issues. First, it may not be possible for two transmissions that use two different spreading codes to occur simultaneously. Obviously, this is a medium access problem. Second, the two transmission can occur simultaneously if the terminals adjust their signal powers so that the interference caused by one transmission is not large enough to destroy packet reception at other terminals. Obviously, this is a power control problem. So the solution to the near-far problem has to have both elements: power control and medium access.

It is important here to differentiate between the spreading code protocol and the MAC protocol. The former decides which PN code is used to spread the signal, but does not solve the contention on the medium. On the other hand, the MAC protocol is responsible for minimizing or eliminating collisions, thereby, achieving good utilization of the available bandwidth. The use of the MAC protocol implies that even if a terminal has an available spreading code, it may not be allowed to transmit.

The design of our MAC protocol, described in detail in subsequent sections, is guided by the following objectives:

- The protocol must be asynchronous, distributed, and scalable for large networks. It must also involve minimal exchange of information and must be suitable for real-time implementation.

- The receiver circuitry should not be overly complex in the sense that it should not be required to monitor the whole code set.

- The protocol should adapt to channel changes and mobility patterns.

- Finally, although we assume that a code assignment protocol is running at a higher layer, the MAC protocol must minimize (or eliminate) collisions even if the code assignment is not "correct". This is important because it is usually difficult to guarantee correct code assignment at all times when network topology is continuously changing.

\subsection{Architecture}

In our design, we use two frequency channels, one for data and one for control (i.e., FDMA-like partitioning). A common spreading code is used by all nodes over the control channel, while several terminal-specific codes can be used over the data channel. This architecture is shown in Figure 4. Note that the different codes used over the data channel are not perfectly orthogonal. However, because of the frequency separation, a signal over the control channel is completely orthogonal to any signal (or code) over the data channel. The splitting of the available bandwidth into two non-overlapping frequency bands is fundamentally needed to allow a terminal to transmit and receive simultaneously over the control and data channels, irrespective of the signal power. As we explain shortly, our protocol utilizes this fact to allow interference-limited transmissions that use (quasiorthogonal) data channel codes to proceed concurrently.

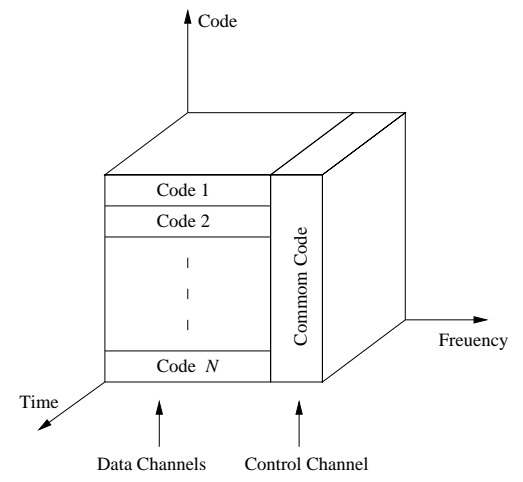

Figure 4: Data and control codes in the proposed protocol.

\subsection{Channel Model and Protocol Assumptions}

In designing our protocol, we assume that: (1) the channel gain is stationary for the duration of the control and the ensuing data packet transmission periods; (2) the gain between two terminals is the same in both directions; and (3) data and control packets between a pair of terminals observe similar channel gains.

In addition to the above assumptions, we assume that the radio interface can provide the MAC layer with the average power of a received control signal as well as the average interference power. Each terminal is equipped with two transceivers and a carrier-sense hardware that senses the control channel for any carrier signal. No carrier-sense is needed for the data channel. The carrier frequency spacing between the control and data channels is enough to ensure that the outgoing signal on one channel does not interfere with the incoming signal on the other channel.

\subsection{Controlled Access CDMA (CA-CDMA) Pro- tocol}

Our CA-CDMA protocol is contention based and uses a modified RTS-CTS reservation mechanism. RTS and CTS packets are transmitted over the control channel (on the common code) at a fixed (maximum) power $P_{\max }$. These packets are received by all potentially interfering nodes, as in the IEEE 802.11 scheme. However, in contrast to the IEEE 802.11 scheme and RA-CDMA protocols, interfering nodes may be allowed to transmit concurrently, depending on some criteria that will be discussed later. For the ensu- 
ing data packet, the receiver and the transmitter must agree on two parameters: the spreading code and the transmission power. Code selection can be done according to any code assignment scheme. As explained later, even if the code assignment scheme is not correct, our protocol will still function properly. The choice of the power level is critical and represents a tradeoff between link quality and MAI. More specifically, as the transmission power increases, the bit error rate at the intended receiver decreases (i.e., link quality improves), but the MAI added to other ongoing receptions increases (i.e., the quality of these receptions deteriorates). In addition to accounting for these two factors, our protocol also incorporates an interference margin in the power computations. This margin allows terminals at some interfering distance from the intended receiver to start new transmissions in the future. The computation of this margin is discussed in Section 4.5.

In the CA-CDMA protocol, terminals exploit knowledge of the power levels of the overheard RTS and CTS messages to determine the power that they can use without disturbing the ongoing receptions. In Section 4.6 we develop a distributed admission control strategy that decides when terminals at some distance can proceed concurrently with their transmissions.

We note here that the CA-CDMA protocol is, to some extent, similar to Qualcomm's CDMA protocol [17], adopted by the US Telecommunication Industry Association as the IS-95 standard for cellular networks. In both protocols, users contend on a control channel to request "network resources". However, the interpretation of "resources" is different in the two protocols; in the Qualcomm protocol, it refers to connection availability, while in the CA-CDMA protocol it refers to a "transmission floor." The similarity is important since the Qualcomm system has proven to be successful.

\subsection{Interference Margin}

An interference margin is needed to allow terminals at some distance from a receiver to start new transmissions in the future. In this section, we describe how this margin is computed. Consider an arbitrary receiver $i$. Let $\mu^{*}$ be the $E_{b} / N_{\text {oeff }}$ ratio that is needed to achieve the target bit error rate at that receiver. It follows from (1) that to achieve the target error rate, we must have

$$
\frac{P_{0}^{(i)}}{P_{\text {thermal }}+P_{\text {MAI }}^{(i)}} \geq \mu^{*}
$$

where $P_{0}^{(i)}$ was defined before, $P_{\text {thermal }}$ is the thermal noise power and $P_{\mathrm{MAI}}^{(i)}$ is the total MAI at receiver $i$ (in (1) $P_{\mathrm{MAI}}^{(i)}=$ $\left.2 \sum_{j=1}^{K} P_{j} / 3 W\right)$. So the minimum required received power is $\left(P_{0}^{(i)}\right)_{\text {min }}=\mu^{*}\left(P_{\text {thermal }}+P_{\text {MAI }}^{(i)}\right)$.

The interference margin strongly depends on the network load, which itself can be conveyed in terms of the so-called noise rise $\left(\xi^{(i)}\right)$, defined as follows: ${ }^{6}$

$$
\xi^{(i)} \stackrel{\text { def }}{=} \frac{\left(\frac{E_{b}}{N_{0}}\right)_{\text {unloaded }}}{\left(\frac{E_{b}}{N_{0}}\right)_{\text {loaded }}}=\frac{P_{\text {thermal }}+P_{\text {MAI }}^{(i)}}{P_{\text {thermal }}}
$$

Note that $\left(P_{0}^{(i)}\right)_{\min }=\xi^{(i)} \mu^{*} P_{\text {thermal }}$ is also dependent

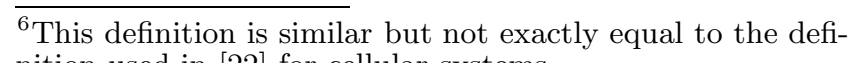
nition used in [22] for cellular systems. on the noise rise. While more capacity can be achieved by increasing the noise rise (i.e., allowing larger $P_{\mathrm{MAI}}^{(i)}$ ), the maximum allowable noise rise is constrained by two factors. First, Federal Communications Commission (FCC) regulations limit the power to some fixed value (e.g., 1 Watt for 802.11 devices). Given this maximum transmission power, as the noise rise is increased, the received power $\left(P_{0}^{(i)}\right)_{\text {min }}$ must increase ( $\mu^{*}$ and $P_{\text {thermal }}$ are constants) and hence, the maximum range (or coverage) for reliable communication will decrease. Second, increasing the noise rise increases the power used to transmit the packet, which in turn increases energy consumption. Energy is a scarce resource in MANETs, so it is undesirable to trade off energy for throughput.

We set the interference margin used by a transmitter to the maximum planned noise rise $\left(\xi_{\max }\right)$, which is obtained by taking into account the above two restrictions on $\xi^{(i)}$. The computations are performed as follows. First, we require that the maximum range, say $d_{\max }$, of our protocol be the same as the maximum range of the 802.11 scheme. For the maximum range, the power used in our protocol equals $\xi^{(i)}$ times the power used in the 802.11 standard. Thus, $\xi_{\max }$ cannot be greater than the ratio of the power limit set by the FCC and the power used in the 802.11 scheme. To account for the second constraint, we choose the interference margin in a manner that maintains the same energy per bit consumed in the 802.11 scheme. The value of the interference margin that achieves the above goals can be derived as follows. We assume that the transmission power attenuates with the distance $d$ as $k / d^{n}$ ( $k$ is a constant and $n \geq 2$ is the loss factor). The minimum required transmit power in CA-CDMA is:

$$
P_{\mathrm{CA}-\mathrm{CDMA}}=\frac{\xi_{\max } \mu^{*} P_{\text {thermal }} d^{n}}{k}
$$

Assuming that the distance $d$ is uniformly distributed from zero to $d_{\max }$, we compute the expectation of $P_{\mathrm{CA}-\mathrm{CDMA}}$ with respect to $d$ :

$$
E\left[P_{\mathrm{CA}-\mathrm{CDMA}}\right]=\frac{\xi_{\text {max }} \mu^{*} P_{\text {thermal }} d_{\max }^{n}}{k(n+1)}
$$

As for the 802.11 protocol, its corresponding transmission power is:

$$
P_{802.11}=\frac{\mu^{*} P_{\text {thermal }} d_{\max }^{n}}{k}
$$

Note that $P_{802.11}$ does not depend on $d$ since the 802.11 standard uses a fixed transmission power.

Accordingly, to achieve equal average energy per bit consumption, we must have:

$$
\frac{E\left[P_{\mathrm{CA}-\mathrm{CDMA}}\right]}{R_{\mathrm{CA}-\mathrm{CDMA}}}=\frac{P_{802.11}}{R_{802.11}}
$$

where $R_{\mathrm{CA}-\mathrm{CDMA}}$ and $R_{802.11}$ are the bit rates for the transmitted data packets in the CA-CDMA and 802.11 protocols, respectively. The reason why these rates can be different is that in our protocol we use two distinct frequency bands, one for control packets and one for data packets, while the standard uses only one band for all packets. Hence, for a fair comparison, data packets in the CA-CDMA protocol must be transmitted at a slower rate. 
From (6), (7), and (8), the interference margin is given by:

$$
\xi_{\max }=(n+1) \frac{R_{\mathrm{CA}-\mathrm{CDMA}}}{R_{802.11}}
$$

As an example, consider the two-ray propagation model with $n=4$, and let the control channel occupy $20 \%$ of the total available bandwidth. Then $\xi_{\max }=6 \mathrm{~dB}$. It is worth noting that $6 \mathrm{~dB}$ lies within the range of values used in already deployed cellular systems [22].

\subsection{Channel Access Mechanism}

We now describe the admission control and channel access strategy in the CA-CDMA protocol. The admission scheme allows only transmissions that cause neither primary nor secondary collisions to proceed concurrently. RTS and CTS packets are used to provide three functions. First, these packets allow nodes to estimate the channel gains between transmitter-receiver pairs. Second, a receiver $i$ uses the CTS packet to notify its neighbors of the additional noise power (denoted by $P_{\text {noise }}^{(i)}$ ) that each of the neighbors can add to terminal $i$ without impacting $i$ 's current reception. These neighbors constitute the set of potentially interfering terminals. Finally, each terminal keeps listening to the control channel regardless of the signal destination in order to keep track of the average number of active users in their neighborhoods. These functions are now explained in detail.

If terminal $j$ has a packet to transmit, it sends a RTS packet over the control channel at $P_{\max }$, and includes in this packet the maximum allowable power level $\left(P_{\text {map }}^{(j)}\right)$ that terminal $j$ can use that will not disturb any ongoing reception in $j$ 's neighborhood. The computation of this power will be discussed shortly. The format of the RTS packet is similar to that of the IEEE 802.11, except for an additional two-byte field that contains the $P_{\text {map }}^{(j)}$ value.

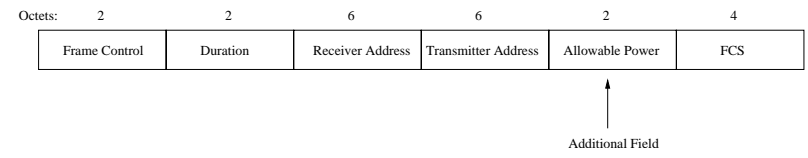

Figure 5: Format of the RTS packet in the CACDMA protocol.

Upon receiving the RTS packet, the intended receiver, say terminal $i$, uses the predetermined $P_{\max }$ value and the power of the received signal $P_{\text {received }}^{(j i)}$ to estimate the channel gain $G_{j i}=P_{\text {received }}^{(j i)} / P_{\max }$ between terminals $i$ and $j$ at that time (note that we assume channel reciprocity, and so $G_{i j}=G_{j i}$ ) - Terminal $i$ will be able to correctly decode the data packet if transmitted at a power $P_{\min }^{(j i)}$ given by:

$$
P_{\text {min }}^{(j i)}=\frac{\mu^{*}\left(P_{\text {thermal }}+P_{\text {MAI-current }}^{(i)}\right)}{G_{j i}}
$$

where $P_{\text {MAI-current }}^{(i)}$ is the effective current MAI from all already ongoing (interfering) transmissions.

Note that because of the assumed stationarity in the channel gain over small time intervals, $G_{j i}$ is approximately constant throughout the transmissions of the control packet and the ensuing data packet. Now, $P_{\min }^{(j i)}$ is the minimum power that terminal $j$ must use for data transmission in order for terminal $i$ to correctly decode the data packet at the current level of interference. This $P_{\min }^{(j i)}$, however, does not allow for any interference tolerance at terminal $i$, and thus all neighbors of terminal $i$ will have to defer their transmissions during terminal $i$ 's ongoing reception (i.e., no simultaneous transmissions can take place in the neighborhood of $i$ ).

Now, according to the link budget calculations in Section 4.5 , the power that terminal $j$ is allowed to use to send to $i$ is given by:

$$
P_{\text {allowed }}^{(j i)}=\frac{\xi_{\max } \mu^{*} P_{\text {thermal }}}{G_{j i}}
$$

If $P_{\text {allowed }}^{(j i)}<P_{\min }^{(j i)}$, then the MAI in the vicinity of terminal $i$ is greater than the one allowed by the link budget. In this case, $i$ responds with a negative CTS, informing $j$ that it cannot proceed with its transmission (the negative CTS is used to prevent multiple RTS retransmissions from $j$ ). The philosophy behind this design is to prevent transmissions from taking place over links that perceive high MAI. This consequently increases the number of active links in the network (subject to the available power constraints).

On the other hand, if $P_{\text {allowed }}^{(j i)}>P_{\text {min }}^{(j i)}$, then it is possible for terminal $i$ to receive $j$ 's signal but only if $P_{\text {allowed }}^{(j i)}$ is less than $P_{\text {map }}^{(j)}$ (included in the RTS). This last condition is necessary so that transmitter $j$ does not disturb any of the ongoing transmissions in its vicinity. In this case, terminal $i$ calculates the interference power tolerance $P_{\text {MAI-future }}^{(i)}$ that it can endure from future unintended transmitters. This power is given by:

$$
P_{\text {MAI-future }}^{(i)}=\frac{3 W G_{j i}}{2 \mu^{*}}\left(P_{\text {allowed }}^{(j i)}-P_{\text {min }}^{(j i)}\right)
$$

Note that the factor $3 \mathrm{~W} / 2$ comes from the spreading gain (see (1)).

The next step is to equitably distribute this power tolerance among future potentially interfering users in the vicinity of $i$. The rational behind this distribution is to prevent one neighbor from consuming the entire $P_{\text {MAI-future }}^{(i)}$ In other words, we think of $P_{\text {MAI-future }}^{(i)}$ as a network resource that should be shared among various terminals. Let $K^{(i)}$ be the number of terminals in the vicinity of $i$ that are

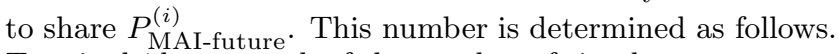
Terminal $i$ keeps track of the number of simultaneous transmissions (i.e., load) in its neighborhood, which we donate by $K_{\text {inst }}^{(i)}$. This can be easily achieved by monitoring the RTS/CTS exchanges over the control channel. In addition, $i$ keeps an average $K_{\mathrm{avg}}^{(i)}$ of $K_{\text {inst }}^{(i)}$ over a specified window. Then, $K^{(i)}$ is calculated as:

$$
K^{(i)}= \begin{cases}\beta\left(K_{\text {avg }}^{(i)}-K_{\text {inst }}^{(i)}\right), & \text { if } K_{\text {avg }}^{(i)}>K_{\text {inst }}^{(i)} \\ \beta, & \text { otherwise }\end{cases}
$$

where $\beta>1$ is a safety margin.

Now, the MAI at terminal $i$ can be split into two components: one that is attributed to terminals that are within the range of $i$ (denoted by $P_{\text {MAI-within }}^{(i)}$ ), and one that is caused by terminals outside that range (denoted by $P_{\text {MAI-other }}^{(i)}$ ). While terminal $i$ can have some control over $P_{\text {MAI-within }}^{(i)}$, it 
cannot influence $P_{\text {MAI-other }}^{(i)}$. We account for this fact in the value of $P_{\text {noise }}^{(i)}$ as follows. In line with cellular systems, we assume that $P_{\text {MAI-other }}^{(i)}=\alpha P_{\text {MAI-within, where } \alpha<1 \text { and }}^{(i)}$ depends mainly on the propagation path loss factor (practical values for $\alpha$ are $\approx 0.5$ for the two-ray model [22]). Accordingly, the interference tolerance $P_{\text {noise }}^{(i)}$ that each future neighbor can add to terminal $i$ is given by

$$
P_{\text {noise }}^{(i)}=\frac{P_{\text {MAI-future }}^{(i)}}{(1+\alpha) K^{(i)}}
$$

When responding to $j$ 's RTS, terminal $i$ indicates in its CTS the power level $P_{\text {allowed }}^{(j i)}$ that $j$ must use. In addition, terminal $i$ inserts $P_{\text {noise }}^{(i)}$ in the CTS packet and sends this packet back to terminal $j$ at $P_{\max }$ over the control channel using the common code. The format of the CTS packet is shown in Figure 6.

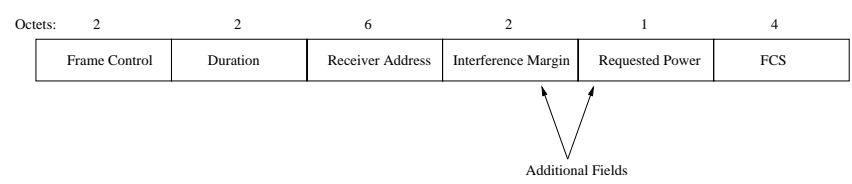

Figure 6: Format of the CTS packet in the proposed protocol.

A potentially interfering terminal, say $s$, that hears the CTS message uses the signal strength of the received CTS to compute the channel gain $G_{s i}$ between itself and terminal $i$. The channel gain along with the broadcasted $P_{\text {noise }}^{(i)}$ values are used to compute the maximum power $P_{\text {map }}^{(s)}$ that $s$ can use in its future transmissions. More specifically, $P_{\text {map }}^{(s)}$ is taken as the minimum of the $P_{\text {noise }}^{(k)} / G_{s k}$ values, for all neighbors $k$ of $s$ (i.e., $P_{\text {map }}^{(s)}$ is updated dynamically whenever $s$ overhears a new CTS). Note that it is possible for more than $K^{(i)}$ terminals to start transmitting during $i$ 's reception and this may result in MAI at $i$ that is greater than $P_{\text {MAI-future }}^{(i)}$ We address this issue in Section 4.7 .

The approach we discussed in this section provides a distributed mechanism for admission control. In contrast to cellular systems where the base station makes the admission decision, in here each terminal, and depending on previously heard RTS and CTS packets, decides whether its transmission can proceed or not.

Following a successful reception of a data packet, receiver $i$ responds with an ACK packet, which is transmitted over the data channel using the same power level that would have been used if $i$ were to send a data packet to $j$. We assume that enough FEC code is used to protect ACK packets from most types of collisions (given the small size of the ACK packets, the FEC overhead is not significant). A similar argument has been used in other, previously proposed protocols (e.g., [21]).

\subsection{Protocol Recovery}

In [7] the authors observed that when the transmission and propagation times of control packets are long, the likelihood of a collision between a CTS packet and a RTS packet of another contending terminal increases dramatically; the vulnerable period being twice the transmission duration of a control packet. At high loads, such a collision can lead to collisions with data packets, as illustrated in Figure 7. Suppose that terminal $D$ starts sending a RTS to terminal $C$ while $C$ is receiving $B$ 's CTS that is intended to $A$. A collision happens at $C$, and hence, $C$ is unaware of $B$ 's subsequent data reception. Afterwards, if $C$ decides to transmit a CTS to $D$, it may destroy $B$ 's reception.

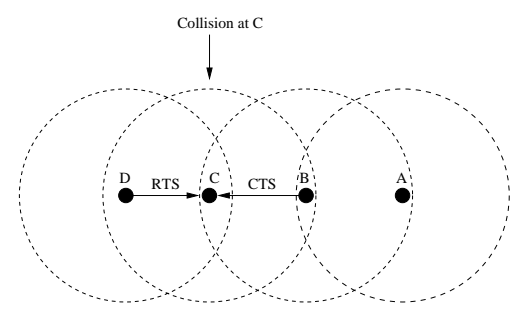

Figure 7: Example of a collision between control packets that eventually leads to a collision with a data packet.

Another problem that was mentioned earlier is if the interference goes above $P_{\text {MAI-future }}^{(.)}$. In CA-CDMA, we avoid the above two problems as follows. Suppose that while receiving a data packet, terminal $i$ hears a RTS message (destined to any terminal) that contains an allowable power $P_{\text {map }}^{(.)}$value that if used could cause an unacceptable interference with $i$ 's ongoing reception. Then terminal $i$ shall respond $i m m e-$ diately with a special CTS packet over the control channel, preventing the RTS sender from commencing its transmission. The duration field of the CTS packet contains the time left for terminal $i$ to finish its ongoing reception. To see how this solution helps in reducing the likelihood of collisions with data packets, consider the situation in Figure 7. Suppose that terminal $A$ sends a RTS to terminal $B$, and $B$ responds back with a CTS that collides at $C$ with a RTS from $D$. Now, $C$ does not know about $B$ 's ongoing reception. Two scenarios can happen. In the first, terminal $C$ may later wish to send a packet to, say, terminal $D$. It sends a RTS, which will be heard by terminal $B$. $B$ responds back with a special CTS. Note that there is a good chance that $B$ 's special CTS will collide with the CTS reply from $D$; however, this is desirable since $C$ will fail to recover $D$ 's CTS packet, and will therefore defer its transmission and invoke its backoff procedure. In essence, $B$ 's special CTS acts as a jamming signal to prevent $C$ from proceedings with its transmission. The second possible scenario is that $D$ (or any other terminal that is out of the maximum range of $B$ ) may send a new RTS to $C$. $C$ will respond to $D$ with a CTS, and $D$ will start sending data to $C$. Simultaneously, $A$ may be sending to $B$, without any collision. This is possible because in CA-CDMA, data and RTS/CTS packets are sent over orthogonal channels.

Note that in CA-CDMA we try to avoid likely collision scenarios such as the one mentioned in [7]. However, there are still few complicated (and definitely much less probable) scenarios where data packets may collide; recovery from such collisions is left to the upper layers. 


\subsection{Code Assignment}

Because of the continuously changing network topology, it is difficult to guarantee correct code assignment at all time. Moreover, since not every node is active at all times, it may be desirable to oversubscribe the medium by assigning the same code to two neighboring terminals, thus violating the assignment goal. In this situation, it is the function of the MAC layer to reduce (or eliminate) contention on the medium. In CA-CDMA, this problem is addressed as follows. When terminal $j$ sends a RTS, it inserts in that RTS the identity of the code that $j$ intends to use for the ensuing data packet. A neighboring terminal that is receiving a packet on the same code can then respond back with the "special" CTS (explained in Section 4.7), which prevents $j$ from commencing its data transmission. Note here the advantage of our architecture, which allows terminals to be informed about all neighborhood activities.

Another possible implementation is to combine the code assignment and access schemes [34]. In such an implementation, the RTS/CTS handshake over the common channel serves to reserve codes so that while the reception is ongoing, no other neighboring terminal can use any of the reserved codes. Although these two problems have been studied separately and dealt with at different layers in the protocol stack, there are two main motivations for combining them. The first is to reduce the overhead of exchanged information sharing. That is, information distributed to solve one problem (e.g., RTS and CTS) can be used to solve the other one (e.g., code assignment). Second, the MAC layer represents the most dynamic and mobility-transparent layer of the protocol stack. Thus, it is beneficial to do code assignment at the MAC layer. On the other hand, separating the two problems has its own advantages, including fairness. It is generally difficult to provide fairness in a contention-based MAC protocol. Thus, an upper layer code assignment can account for that.

\section{PROTOCOL EVALUATION}

\subsection{Simulation Setup}

We now evaluate the performance of the CA-CDMA protocol and contrast it with the IEEE 802.11 scheme. Our results are based on simulation experiments conducted using CSIM programs (CSIM is a C-based process-oriented discrete-event simulation package). In our simulations, we investigate both the network throughput as well as the energy consumption. For simplicity, data packets are assumed to have a fixed size. Each node generates packets according to a Poisson process with rate $\lambda$ (same for all nodes). The routing overhead is ignored since the goal here is to evaluate the performance improvements due to the MAC protocol. Furthermore, because the interference margin is chosen so that the maximum transmission range under the CA-CDMA and 802.11 protocols is the same, it is safe to assume that both protocols achieve the same forward progress per hop. Consequently, we can focus on the one hop throughput, i.e., the packet destination is restricted to one hop from the source. The random waypoint model is used for mobility, with a host speed that is uniformly between 0 and 2 meters/sec. Note, however, that mobility has a little effect on our protocol, since an RTS-CTS exchange preceeds ev- ery packet transmission. The transmission periods for the RTS, CTS, data, and ACK packets are all in tens of milliseconds, so no significant changes in topology take place within these periods. The capture model is similar to the one in [32]. Other parameters used in the simulations are given in Table 1. These paraments correspond to realistic hardware settings [1].

Table 1: Parameters used in the simulations.

\begin{tabular}{|c|c|}
\hline Data packet size & $2 \mathrm{~KB}$ \\
\hline 802.11 data rate & $2 \mathrm{Mbps}$ \\
\hline CA-CDMA data rate & $1.6 \mathrm{Mbps}$ \\
\hline Control channel rate & $400 \mathrm{Kbps}$ \\
\hline Processing gain & 11 \\
\hline SNR threshold & $10 \mathrm{~dB}$ \\
\hline Reception threshold & $-94 \mathrm{dBm}$ \\
\hline Carrier-sense threshold & $-108 \mathrm{dBm}$ \\
\hline Thermal+receiver noise & $-169 \mathrm{dBm} / \mathrm{Hz}$ \\
\hline 802.11 power & $20 \mathrm{dBm}$ \\
\hline$\xi_{\max }$ & $6 \mathrm{~dB}$ \\
\hline
\end{tabular}

\subsection{Simulation Results}

We consider two types of topologies: random grid and clustered. In the random grid topology, $M$ mobile hosts are placed across a square area of length 3000 meters. The square is split into $M$ smaller squares. The location of a mobile user is selected randomly within each of these squares. For each generated packet, the destination node is randomly selected from the one-hop neighbors.

The performance for random grid topologies is demonstrated in Figure 8. In parts (a) and (b), we set $M=36$ and vary the packet generation rate $(\lambda)$. Part (a) of the figure depicts the network throughput. It is shown that CACDMA achieves up to $280 \%$ increase over the throughput of the IEEE 802.11 scheme. This increase is attributed to the increase in the number of simultaneous transmissions. Furthermore, CA-CDMA saturates at about twice the load at which the 802.11 scheme saturates.

Part (b) of Figure 8 depicts the energy consumption versus $\lambda$. Energy consumption is the total energy used to successfully transmit a packet. It includes the energy of the control packets and the lost energy in retransmitting data and control packets in case of collisions. For almost all cases, CA-CDMA requires less than $50 \%$ of the energy required under the 802.11 scheme. This may, at first, seem to counterintuitive, since in Section 4.5 the interference margin was chosen so that both protocols consume the same energy per packet. However, according to the topology we examine here, the transmitter-receiver separation distance is not uniform. More links are formed with neighbors that are much closer than the maximum transmission range (1061 meters in our simulations). Unlike the 802.11 scheme, CA-CDMA makes use of shorter links to save energy. Note that in both protocols, the required energy increases with the load. The reason for this is that as $\lambda$ increases, the probability of collisions also increases, and hence, more energy has to be spent on retransmissions.

In Part (c) of Figure 8 we investigate the effect of varying the number of nodes while the dimensions of the region are kept fixed $(3000 \mathrm{~m} \times 3000 \mathrm{~m})$. Persistent load is used in this experiment, i.e., nodes always have packets to send. As 


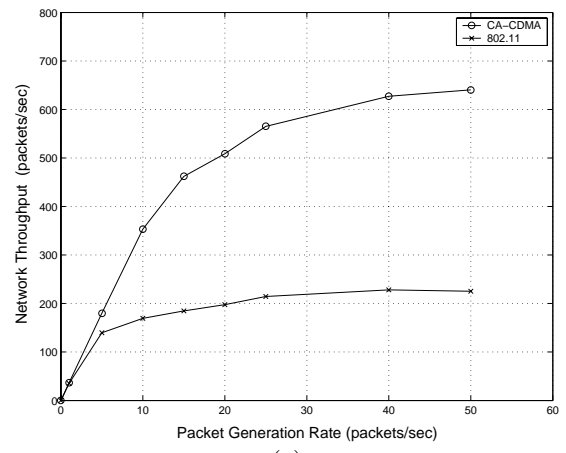

(a)

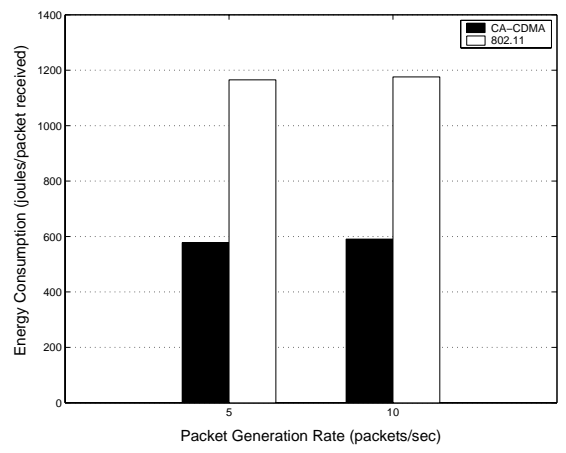

(b)

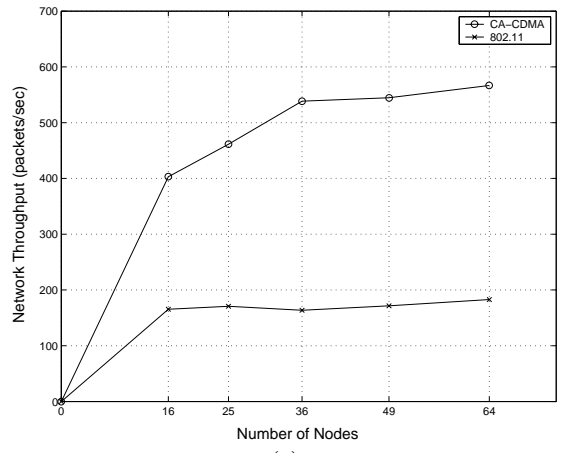

(c)

Figure 8: Performance of the CA-CDMA and the 802.11 protocols (random grid topologies).

shown in the figure, the throughput enhancement due to CACDMA increases with node density. This can be explained by noting that CA-CDMA bounds the transmission power rather that prevents simultaneous transmissions. Therefore, as the density of nodes increases, more concurrent links are formed and the network throughput increases. The 802.11 scheme reserves a fixed floor, and thus, all nodes within that floor have to defer their transmissions. Therefore, the density of the nodes has little effect on the 802.11 throughput.

The authors in [20] argued that traffic locality is the key factor in determining the feasibility of large ad hoc networks. This motivates studying the performance of CA-CDMA under clustered topologies. In such topologies, a node communicates mostly with nodes within its own cluster, and rarely with neighboring cluster nodes. These topologies are common in practice (e.g., a historical site where users of wireless devices move in groups). To generate a clustered topology, we consider an area of dimensions $1000 \times 1000$ (in meters). We let $M=24$ nodes, which are split into 4 equal groups, each occupying a $100 \times 100$ square in one of the corners of the complete area. For a given source node, the destination is selected from the same cluster with probability $1-p$ or from a different cluster with probability $p$. In each case, the selection from within the given cluster(s) is done randomly.

Part (a) of Figure 9 depicts the network throughput versus $\lambda$ for $p=0.25$. According to the 802.11 scheme, only one transmission can proceed at a time since all nodes are within the carrier-sense range of each other. However, according to CA-CDMA, three to four transmissions can proceed simultaneously, resulting in a significant improvement in network throughput. In Part (b) of the figure, we further investigate the locality of the traffic by fixing $\lambda$ and varying $p$. Indeed, as the figure shows, the locality of the traffic can highly impact the network throughput of CA-CDMA, while the 802.11 performance is almost unchanged. As the traffic locality increases (i.e., $p$ decreases) the enhancement of CA-CDMA increases.

\section{CONCLUSIONS AND FUTURE WORK}

In this paper, we proposed a CDMA-based power controlled MAC protocol for wireless ad hoc networks. This protocol, called CA-CDMA, accounts for the multiple access interference, thereby solving the near-far problem that undermines the throughput performance in MANETs. CA-
CDMA uses channel-gain information obtained from overheard RTS and CTS packets over an out-of-band control channel to dynamically bound the transmission power of mobile terminals in the vicinity of a receiver. It adjusts the required transmission power for data packets to allow for interference-limited simultaneous transmissions to take place in the neighborhood of a receiving terminal.

We compared the performance of our protocol with that of the IEEE 802.11 scheme. Our simulation results showed that CA-CDMA can improve the network throughput by up to $280 \%$ and, at the same time, achieve $50 \%$ reduction in the energy consumed to successfully deliver a packet from the source to the destination. To the best of our knowledge, CA-CDMA is the first protocol to provide a solution to the near-far problem in CDMA ad hoc systems at the protocol level.

Our future work will focus on other capacity optimizations such as the use of directional antennas in CDMA-based protocols. Because of MAI effects, CDMA benefits significantly from smart antennas. Variable rate support is another optimization that we have not considered in this work. In [13] the authors showed that adapting the transmit power, data rate, and coding scheme achieves maximum spectral efficiency. The 802.11 scheme allows nodes to increase their information rate up to $11 \mathrm{Mbps}$ when the power at the receiver is far more than necessary to achieve $2 \mathrm{Mbps}$. It could be possible to improve the proposed scheme by increasing the information rate (i.e, decreasing the prossing gain) when the MAI is much less than the planned interference margin (i.e., load is low). This is desirable to allow the developed protocol to adapt to different working conditions in terms of the load offered by the users in the network.

\section{REFERENCES}

$[1]$ http://www.cisco.com/warp/public/cc/pd/witc/ao350ap.

[2] http://www.ietf.org/html.charters/manetcharter.html.

[3] International Standard ISO/IEC 8802-11; ANSI/IEEE Std 802.11, 1999 Edn. Part 11: wireless LAN Medium Access Control (MAC) and Physical Layer (PHY) specifications.

[4] A. A. Bertossi and M. A. Bonuccelli. Code assignment for hidden terminal interference avoidance in multihop 


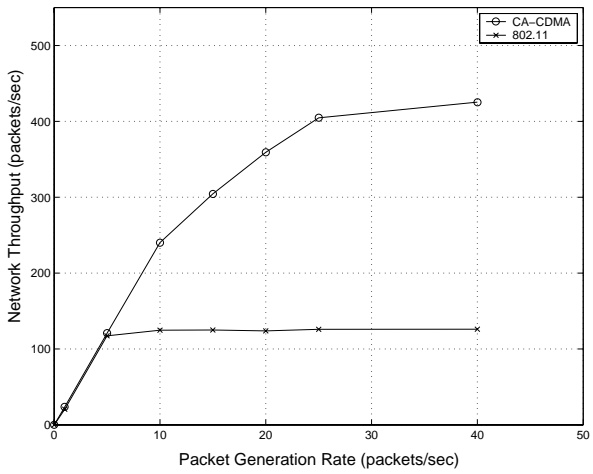

(a)

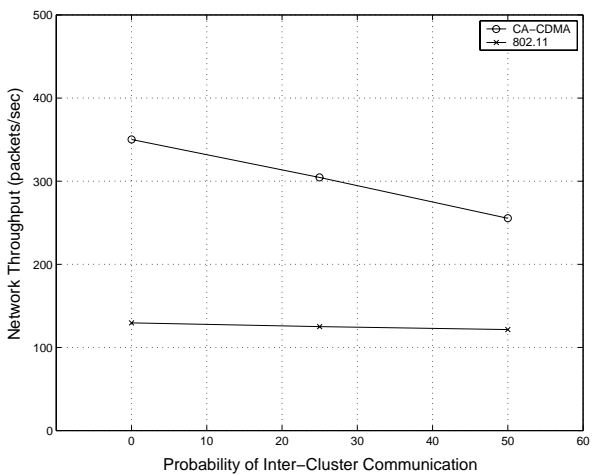

(b)

Figure 9: Performance of the CA-CDMA and 802.11 protocols as a function of $\lambda$ (clustered topologies).

packet radio networks. IEEE Transactions on Communications, 3(4):441-449, Aug. 1995.

[5] M.-S. Chen and R. Boorstyn. Throughput analysis of code division multiple access (CDMA) multihop packet radio networks in the presence of noise. In Proceedings of the IEEE INFOCOM Conference, pages 310-316, 1985.

[6] I. Cidon and M. Sidi. Distributed assignment algorithms for multihop packet radio networks. IEEE Transactions on Computers, 38(10):1353-1361, Oct. 1989.

[7] J. Deng and Z. Haas. Dual busy tone multiple access (DBTMA): A new medium access control for packet radio networks. In Proceedings of the IEEE ICUPC, pages 973-977, Oct. 1998.

[8] O. deSouza, M.-S. Chen, and R. R. Boorstyn. A comparison of the performance of protocols in pakcet radio networks. In Proceedings of the IEEE MILCOM Conference, volume 2, pages 455-460, Oct 85.

[9] T. ElBatt and A. Ephremides. Joint scheduling and power control for wireless ad-hoc networks. In Proceedings of the IEEE INFOCOM Conference, volume 2, pages 976-984, 2002.

[10] A. Ephremides and T. V.Truong. Scheduling broadcasts in multihop radio networks. IEEE Transactions on Communications, 38(4):456-460, Apr. 1990.

[11] J. Garcia-Luna-Aceves and J. Raju. Distributed assignment of codes for multihop packet-radio networks. In Proceedings of the IEEE MILCOM Conference, volume 1, pages 450-454, 1997.

[12] K. S. Gilhousen, I. M. Jacobs, R. Padovani, A. J. Viterbi, L. A. Weaver, and C. E. W. III. On the capacity of a cellular CDMA system. IEEE Transactions on Vehicular Technology, 40:303-312, May 1991.

[13] A. Goldsmith and P. Varaiya. Increasing spectral efficiency through power control. In Proceedings of the IEEE ICC Conference, pages 600 - 604, 1993.

[14] L. Hu. Distributed code assignments for CDMA packet radio networks. IEEE/ACM Transactions on Networking, 1:668-677, Dec. 1993.
[15] K.-W. Hung and T.-S. Yum. The coded tone sense protocol for multihop spread-spectrum packet radio networks. In Proceedings of the IEEE GLOBECOM Conference, volume 2, pages 712-716, 1989.

[16] M. Joa-Ng and I.-T. Lu. Spread spectrum medium access protocol with collision avoidance in mobile ad-hoc wireless network. In Proceedings of the IEEE INFOCOM Conference, volume 2, pages 776-783, 1999.

[17] R. Kerr. CDMA digital cellular: An ASIC overview. In Appl. Microwave and Wireless, pages 30-41, Fall 1993.

[18] T. J. Kwon and M. Gerla. Clustering with power control. In Proceedings of the IEEE MILCOM Conference, volume 2, pages 1424-1428, 1999.

[19] S. W. Lee and D. H. Cho. Distributed reservation CDMA for wireless lan. In Proceedings of the IEEE GLOBECOM Conference, volume 1, pages 360-364, 1995.

[20] J. Li, C. Blake, D. S. Couto, H. I. Lee, and R. Morris. Capacity of ad hoc wireless networks. In Proceedings of the IEEE/ACM MOBICOM Conference, pages 61-69, 2001.

[21] J. Monks, V. Bharghavan, and W.-M. Hwu. A power controlled multiple access protocol for wireless packet networks. In Proceedings of the IEEE INFOCOM Conference, volume 1, pages 219-228, 2001.

[22] T. Ojanperä and R. Prasad. Wideband CDMA For Third Generation Mobile Communications. Artech House, Incorporated, 1998.

[23] R. L. Pickholtz, D. L. Schilling, and L. B. Milstein. Theory of spread spectrum communications-a tutorial. IEEE Transactions on Communications, 30:855-884, May 1982.

[24] J. G. Proakis. Digital Communications. McGraw-Hill, Inc., 2001.

[25] M. B. Pursley. Performance evaluation for phase-coded spread-spectrum multiple-access commuincation-part 1: System analysis. IEEE Transactions on Communications, 25(8):795-799, Aug. 1977.

[26] R. Ramanathan and J. Redi. A brief overview of ad hoc networks: challenges and directions. IEEE Communications Magazine, 40(5):20-22, May 2002. 
[27] T. Rappaport. Wireless Communications: Principles and Practice. Prentice Hall, 2002.

[28] V. Rodoplu and T. Meng. Position based CDMA with multiuser detection (P-CDMA/MUD) for wireless ad hoc networks. In IEEE Sixth International Symposium on Spread Spectrum Techniques and Applications, volume 1, pages 336-340, 2000.

[29] M. K. Simon, J. K. Omura, R. A. Scholtz, and B. K. Levitt. Spread Spectrum Communications Handbook. McGraw-Hill, Inc., 1994.

[30] E. Sousa and J. A. Silvester. Spreading code protocols for distributed spread-spectrum packet radio networks. IEEE Transactions on Communications, 36(3):272-281, Mar. 1988.

[31] E. Sousa and J. A. Silvester. Optimum transmission ranges in a direct-sequence spread-spectrum multihop packet radio network. IEEE Journal on Selected Areas in Communications, 8(5):762-771, June 1990.
[32] C. Ware, J. Chicharo, and T. Wysocki. Simulation of capture behaviour in IEEE 802.11 radio modems. In Proceedings of the IEEE Vehicular Tech. Conference, volume 3, pages 1393-1397, Fall 2001.

[33] J. E. Wieselthier, A. Ephremides, and J. A. B. Tarr. A distributed reservation-based CDMA protocol that does not require feedback information. IEEE Transactions on Communications, 36(8):913-923, Augut 1988.

[34] S.-L. Wu, C.-Y. Lin, Y.-C. Tseng, and J.-P. Sheu. A new multi-channel mac protocol with on-demand channel assignment for multi-hop mobile ad hoc networks. In Proceedings of the International Symposium on Parallel Architectures, Algorithms and Networks, pages 232-237, 2000. 